\title{
Simulating Structural Response to Water Impact
}

\author{
J.C. Campbell*, R. Vignjevic \\ School of Engineering, Cranfield University, Cranfield, Bedfordshire, MK43 OAL. UK \\ * Corresponding author: Phone: +44 1234 754613, e-mail: j.campbell@cranfield.ac.uk
}

\begin{abstract}
Structural response to water impact is important for several areas, including the aerospace and marine industries. Aircraft must be designed to cope with ditching and offshore structures are subject to extreme wave impact and green water loading. The goal is a reliable technique for predicting the structural response to extreme water loading. This is a complex problem involving the interaction of non-linear fluid behaviour (breaking waves, fluid impact) with non-linear structural behaviour (large deformations, contact, material plasticity and damage). This paper gives an overview of the coupled FE/SPH approach for modelling water impact on structures and discusses specific issues related to the analysis of floating structures. The capabilities of the method are illustrated first through comparison with published numerical results for simple problems involving the equilibrium of rigid floats and an initially submerged cylindrical float. Then by comparison with experimental data for the collapse of a metallic helicopter floor structure due to impact with water. Finally the response of a moored buoy to an extreme wave is modelled. The sensitivity of the model to spatial resolution is investigated for the rising cylinder and helicopter floor cases.
\end{abstract}

Keywords: Smoothed Particle Hydrodynamics, coupled SPH-FE, fluid-structure interaction, extreme wave impact 


\section{Introduction}

Extreme waves are a natural phenomenon that can cause significant damage to ships and offshore structures, in particular through green-water loading. The term green water indicates the flow of water, not just spray, over the deck of an offshore structure. This type of fluid-structure interaction is a potentially highly complex non-linear problem that can combine several features:

- The behaviour of extreme waves, including breaking,

- The response of a floating structure to waves,

- The interaction of the water with a structure,

- The response of the structure to the fluid loading including large deformations and non-linear material behaviour (including plasticity, damage and failure).

The absence of reliable theoretical models for this behaviour means that design must be based on empirical considerations and model test data.

The objective of this research is to develop and demonstrate an analysis tool capable of predicting the response of structures to extreme wave loading. To achieve this objective the tool must be capable of representing the important feature of the problem. The explicit finite element (FE) method is solidly established for the simulation of structural response and is well suited to representing the types of structural deformation that can occur in water impact. However due to the well known problems with mesh-tangling this method is not appropriate for modelling the fluid. In order to simulate the structural response to extreme wave loading the FE method must be coupled with another method appropriate for modelling the fluid behaviour. In this work we have used the Smoothed Particle Hydrodynamics (SPH) method to model the fluid behaviour as it can be easily and reliably coupled with the nonlinear FE method [1]. This is an established approach that has been successfully used to study the impact of rigid [2] and elastic[3] shapes with water as well as the response of 
floating rigid objects to waves [3,4]. For the impact of rigid shapes with water [2], Anglileri et al. show good correlation between simulation and experiment for accelerations and impact pressures.

The SPH method is one of the earliest developed and widely used particle methods. $\mathrm{SPH}$ was initially developed for the simulation of astrophysics problems $[5,6]$, with the critical development being a method for the calculation of derivatives without a computational mesh. The absence of a mesh allows large material deformations to be modelled without numerical problems. The application of SPH to water waves and related free-surface hydrodynamics problems was begun by Monaghan [7], who performed twodimensional simulations of a dam break problem and wave propagation onto a shallow beach. More recently SPH simulations have been further compared with published experimental results, an example being Scott Russell's wave generator [8], with the SPH method in agreement with the experimental results. In these simulations use an artificial equation of state to produce a quasi-incompressible fluid, the approach used in this work. SPH has also been used for wave mechanics with exact enforcement of incompressibility (ISPH) [9]. This uses an implicit pressure update that allows a larger time step but requires more computational work per time step. A comparison of the two approaches by Lee et al. [10] concludes that overall ISPH yields a more reliable result. A more recent study by Hughes and Graham [11] concludes that, with a suitable formulation, compressible SPH performs at least as well as ISPH.

SPH methods have been successfully applied to $2 \mathrm{D}$ simulations of green water overtopping [12] and wave overtopping [13] using rigid representations of the impacted structure. Three-dimensional fluid models often require significant computational resources due to the number of particles required to obtain adequate resolution. While the examples shown in this paper all used a single processor, parallel versions of SPH have existed for 
many years [14] and today SPH codes exist for astrophysics [15] and for fluid behaviour $[16,17]$ that are able to effectively use a large number of cores to analyse large problems including test problems in excess of 100 million particles.

SPH has been applied to a number of free-surface slow and maritime applications. More information on the SPH method and applications can be found through the SPH European Research Interest Community (SPHERIC) website [18], which includes an extensive list of SPH related references.

Other approaches for wave impact simulations typically use Eulerian or ALE methods to solve the Navier-Stokes equations. For example, Buchner, Kleefsman and coworkers $[19,20]$ have developed an Eulerian code and applied it to wave run-up and green water loading on Floating Production Storage and Offloading (FPSO) systems. Their results show good agreement with experiment for structural loads, however the structures are represented by rigid shapes.

Previous work at Cranfield has developed and demonstrated a contact algorithm for coupling meshless and finite element discretisations, allowing complex interaction in three dimensional simulations [1]. A primary application of this method was the simulation of helicopter crash on water and it has been demonstrated for the impact of rigid and deformable structures on water [21]. In addition kernel interpolation [22], including normalized corrected kernels, and rigorous derivation of the SPH equations [23] have been investigated. All developments have been implemented and tests within the 3D SPH solver developed at Cranfield. This solver is directly linked to the LLNL-DYNA3D explicit non-linear FE code [24] to allow coupled FE/SPH analysis. Extension of this tool to wave loading of offshore structures required development and implementation of appropriate initial and boundary conditions for the fluid component. 


\section{Overview of SPH and coupling}

The SPH method is based on an interpolation technique that allows any physical field (scalar, vector or tensor) $f(\mathbf{x})$ to be approximated by a smoothed value $\langle f(\mathbf{x})>$

$$
\langle f(\mathbf{x})\rangle=\int_{\Omega} f(\mathbf{s}) W(\mathbf{x}-\mathbf{s}, h) d s,
$$

where $f$ is a function of position vector $\mathbf{x}$ defined in the domain $\Omega$. The kernel function, $W(\mathbf{x}-\mathbf{s}, h)$, is centered at point $\mathbf{x}$ and is a function of position. The smoothing length, $h$, controls the extent of the domain where $W$ is non-zero, usually defined in three dimensions as a sphere of radius $2 h$. The kernel function must satisfy two conditions, that $W$ becomes the delta function $\delta$ in the limit $h \rightarrow 0$ and that

$$
\int_{\Omega} W(\mathbf{x}-\mathbf{s}, h) d s=1 .
$$

i.e. the kernel functions represent a partition of unity.

The most commonly used form of the kernel function in SPH is the B-Spline kernel:

$$
W(\mathbf{x}-\mathbf{s}, h)=C\left\{\begin{array}{cc}
1-\frac{3}{2} z^{2}+\frac{3}{4} z^{3}, & \text { if } 0 \leq z<1 \\
\frac{1}{4}(2-z)^{3}, & \text { if } 1 \leq z<2 \\
0 . & \text { otherwise }
\end{array}\right.
$$

Where $z$ is the ratio of the length $\mathbf{x}-\mathbf{s}$ and the smoothing length $h$. This is the form of the kernel used in this work.

The gradient of function $f$ can then be approximated by using equation 1 , which becomes, following integration by parts and neglecting surface terms due to the compact support of the kernel:

$$
\langle\nabla f(\mathbf{x})\rangle=\int_{\Omega} f(\mathbf{s}) \nabla W(\mathbf{x}-\mathbf{s}, h) d s
$$


This continuous integral must now be written as a discrete approximation for the case where the physical field is only known at a set of $N$ discrete points, known in SPH as particles. Equation 4 is discretised as follows

$$
\langle\nabla f(\mathbf{x})\rangle_{i} \approx \sum_{j=1}^{N} V_{j} f\left(\mathbf{x}_{j}\right) \nabla W\left(\mathbf{x}_{i}-\mathbf{x}_{j}, h\right),
$$

here $V_{j}$ is the volume of particle $j$, usually defined as $m_{j} / \rho_{j}$, where $m_{j}$ and $\rho_{j}$ are the mass and density respectively of particle $j$. The SPH method thus uses a set of nodes, or particles, to represent a material. The field values, such as density and pressure, are known at the particles which move with the material. During a calculation the set of particles within the support of particle $i$ can change, allowing large material deformations to be represented without numerical problems.

Applying this process to the momentum equation of continuum mechanics

$$
\frac{d \mathbf{v}}{d t}=-\frac{1}{\rho} \nabla P
$$

allows the common SPH form of the momentum equation to be derived,

$$
\frac{d \mathbf{v}_{i}}{d t}=-\sum_{j} m_{j}\left[\frac{P_{i}}{\rho_{i}^{2}}+\frac{P_{j}}{\rho_{j}^{2}}\right] \nabla_{i} W_{i j} .
$$

Where $P_{i}$ and $P_{j}$ are the pressure at particle $i$ and $j$ respectively, and $\mathbf{v}$ is the velocity vector.

A consequence of the SPH method is that the location of the boundary of a solid or liquid is not well defined. Each particle is the centre of a sub-domain with radius $2 h$ and consequently the boundary is diffused over this length. To avoid the requirement to construct a surface geometry from the SPH nodes a contact potential, $\phi_{x}$, based on the kernel function is used [1]. This potential is defined as

$$
\phi_{x}\left(\mathbf{x}_{i}\right)=\int_{\Omega_{c}} K\left(\frac{W\left(\mathbf{x}_{i}-\mathbf{x}_{j}, h\right)}{W\left(\Delta p_{\text {avg }}\right)}\right)^{n} d V
$$


where $\mathbf{x}_{i}$ and $\mathbf{x}_{j}$ are the coordinates of two particles in different bodies, $\Delta p_{\text {avg }}$ denotes the average node spacing in the neighbourhood of particle $i$. $K$ and $n$ are user defined parameters that control the magnitude of the potential. A discrete form of this contact potential is then derived and used to define the contact force, $\mathbf{f}_{i}$ :

$$
f_{i}=\frac{m_{i}}{\rho_{i}} \sum_{j} \frac{m_{j}}{\rho_{j}} K n \frac{W\left(x_{i}-x_{j}, h\right)^{n-1}}{W\left(\Delta p_{\text {avg }}\right)^{n}} \nabla W\left(x_{i}-x_{j}, h\right) .
$$

In applying this contact potential to contact between $\mathrm{FE}$ and $\mathrm{SPH}$, the contact force is applied between an SPH particle and FE node that lies within $2 h$ of that particle [1], see Fig. 1.

\section{Water modelling with SPH}

Simulation of wave loading requires significantly more complex initial and boundary conditions for the water component than required for the impact of a structure on water considered previously [1,21].

At the start of the simulation the problem must be under static equilibrium. If it is not the initial behaviour of the model will be governed by this lack of equilibrium, potentially obscuring the behaviour of interest. The initial equilibrium must be determined for

- $\quad$ Pressure vs. depth in the water

- Initial contact forces between structure and water. This is particularly critical for floating bodies.

The pressure-depth relationship can de determined analytically for a given equation of state, therefore it is possible to determine the correct density for each particle. The initial contact forces can not be determined analytically due to its non-linear dependence on the relative positions of the SPH particles and FE nodes. Consequently a numerical technique was required to determine the initial state of a problem. 
The dynamic relaxation method was selected to solve the initial static problem. Dynamic relaxation is a commonly used method that allows an explicit transient solver to approximate the solution to a static problem, see for example the DYNA3D implementation [24]. The existing dynamic relaxation algorithm in DYNA3D was extended to include the SPH solver. Due to the computational cost of the dynamic relaxation solution on 3D coupled simulations the coupled solver was extended to allow the state at the end of the dynamic relaxation simulation to be saved to a file and later used as the initial condition of a transient simulation.

Figure 2 shows the initial and final states of a dynamic relaxation simulation of a 2D simulation to determine the floating equilibrium of a rigid rectangle with a density of half the water density. During the dynamic relaxation process the rotation of the rectangular float was prevented. The final state shows the correct pressure depth profile in the water and contact equilibrium between the SPH water and FE float.

Under the conditions of wave loading the water can be considered incompressible. The SPH solver used assumes that all materials are compressible and therefore a bulk stiffness, in the form of an equation of state (EOS), must be defined. For free surface flows and water impact simulations the Murnaghan EOS is commonly used for the water. This has the form

$$
P=B\left[\left(\frac{\rho}{\rho_{0}}\right)^{\gamma}-1\right],
$$

where $B$ and $\gamma$ are user supplied material coefficients. For an incompressible fluid the parameter B can be chosen to give an artificially low speed of sound, while keeping the density changes small. This is valid provided the flow velocities are small compared with the speed of sound [7]. The advantage of this is that a lowered speed of sound increases the critical time step size for the explicit time integration algorithm, potentially reducing the CPU 
cost of a simulation. However when linked with a deformable structural model, the critical time-step size is often controlled by the structural model as the speed of sound in the structural material is much higher than in the water; for example the speed of sound in steel is approximately $4,500 \mathrm{~m} / \mathrm{s}$ against $1,500 \mathrm{~m} / \mathrm{s}$ for water. Consequently there is often no practical advantage in computational cost in using an artificial speed of sound once a deformable structural model is included in the analysis.

\section{Numerical demonstration cases}

A set of numerical experiments have been performed to confirm the capability of the coupled FE-SPH approach to correctly represent the behaviour of floating bodies and water interaction with deformable structures.

\subsection{Equilibrium of floating objects}

A useful verification of the method's ability to simulate the response of a floating body is to demonstrate that it will reach its correct equilibrium state. Three test cases were selected for which published results [25], are available. The published results were obtained using an Eulerian solver coupled with rigid shapes.

In the first case a $10 \mathrm{~cm}$ square rigid float, with density half the density of the fluid, is in initial unstable equilibrium, fig. 3a. As the float is rigid, the Murnaghan EOS is used in these analyses. Dynamic relaxation has been used to determine the initial pressure and contact equilibrium using additional constraints to prevent rotation of the float. In the simulation the float rotates through 45 degrees to its position of stable equilibrium, fig. $3 \mathrm{~b}$, and settles in that state after a period of damped oscillation. A comparison between the current results and the Eulerian results [25] is shown in fig. 4, showing good agreement. The zero time for the current results has been shifted so that the time of initial rotation agrees as 
the published results used an initial rotational velocity as a disturbance, while no initial disturbance was used in the current work.

A second test was run using the square float, with the density of the square reduced to one quarter that of the fluid. The initial conditions of the simulation were identical to the half density case, so the float will initially rise before settling down to its stable equilibrium position, fig. 5. For this problem the theoretical value for the final angle of rotation is 63.4 degrees. In the simulation the float, after oscillation, settles down to approximately 63 degrees, fig. 6.

The third test used a $10 \mathrm{~cm}$ by $5 \mathrm{~cm}$ rectangular float with density half the density of the fluid. The initial position of the float is with the long axis vertical, with the initial unstable equilibrium determined by a dynamic relaxation simulation to determine the pressure and contact equilibrium, fig. 2. In the simulation the float rotates to the stable equilibrium position with the long axis horizontal, figures 7 and 8.

These results from these tests show that the coupled FE-SPH approach will correctly predict the equilibrium floating behaviour of a floating body.

\subsection{Rising cylinder}

In the final $2 \mathrm{D}$ test presented in this paper, a cylinder of radius $1 \mathrm{~m}$ is initially submerged and is released to rise to the surface. The cylinder has density 0.6 times the fluid density and the centre of mass is initially at a depth of $5 \mathrm{~m}$. This test case is used for Eulerian codes to demonstrate the ability of a shape to break through a free surface. This is not an issue for the SPH method as the location of the free surface is determined naturally by the particle positions and does not require special treatment.

For our analysis the channel width has been set to $6 \mathrm{~m}$ and the initial depth of water to $6.9 \mathrm{~m}$. Analyses have been run for three different initial particle sizes: $0.1 \mathrm{~m}, 0.05 \mathrm{~m}$ and 
0.025m. Equating to 3650,15492 and $60932 \mathrm{SPH}$ particles respectively. Dynamic relaxation was used to determine the initial conditions for all the simulations.

The in each analysis the cylinder rises through the fluid, figure 9a, and then breaks through the fluid surface, figure $9 \mathrm{~b}$. The results for the cylinder velocity and centre-point displacement, figures $10 \mathrm{a}$ and $10 \mathrm{~b}$, show little difference until the cylinder is close to the surface. It can then be seen that the maximum velocity decreases with the initial particle spacing from $2.5 \mathrm{~m} / \mathrm{s}$ for the low resolution model to $2.35 \mathrm{~m} / \mathrm{s}$ for the highest. Overall the correlation with the published Eulerian results [25] is reasonable for all models, with the peak velocity for the highest resolution model giving the best agreement. The results show the cylinder rising through the fluid, figure $9 \mathrm{a}$, and then breaking through the fluid surface, figure 9b. These results show that the coupled approach can correctly treat the buoyancy of a submerged object, with even a small number of particles providing reasonable behaviour.

\subsection{Impact of a deformable structure with water}

As discussed earlier a specific strength of the coupled FE-SPH approach is that is allows for fluid-structure interaction even when the structure is undergoing large non-linear deformation. The availability of appropriate tests cases for this behaviour is limited, so for this work the impact of a metallic helicopter sub-floor structure with water has been selected as experimental data was available.

The experiment was performed under the CAST (Crashworthiness of Helicopters on Water) EU Framework 5 project [26]. The structure was manufactured from aluminium and designed to be characteristic of a metallic helicopter cabin sub-floor structure. In the experiment the structure was mounted on a trolley and dropped vertically onto water with an impact velocity of $7.95 \mathrm{~m} / \mathrm{s}$. The total mass on the structure and trolley was approximately $600 \mathrm{~kg}$. 
A finite element model of the sub-floor structure was constructed using shell elements with appropriate elastic-plastic material properties. The trolley was represented as a rigid mass, and time history information for the trolley acceleration was measured. The behaviour of the structural model was verified against experimental results for impact with a rigid surface. While the time-step size is controlled by the structural model, the cost per step of the SPH fluid model is significant and parametric studies were performed to investigate the effect of particle size, the depth of the water domain and the distance from the structure to the edge of the water domain.

Three particle sizes were investigated for a water domain of $2.44 \mathrm{~m} \times 1.68 \mathrm{~m} \times$ 0.96m: $5 \mathrm{~cm}$ (31008 particles), $4 \mathrm{~cm}$ (61488 particles) and 3cm (143,360 particles). The size of the domain for this study was chosen from previous experience. The models took about 18 hours to run for the coarse model, about 35 hours for the medium and 78 hours for the fine model. In all cases the critical time step size for the analysis was limited by the structural model. The initial state of the medium model is shown in fig. 11, the structure consists of approximately 38000 shell elements. The trolley accelerations for each model are shown in fig. 12. There is a clear difference between the coarse model and the two finer models, while the medium and fine models show much better correlation. Based on this the $4 \mathrm{~cm}$ particle size was used for the further studies.

A water domain of $2.44 \mathrm{~m} \times 1.68 \mathrm{~m}$ gives a distance of approximately $0.95 \mathrm{~m}$ from the edge of the structure to the edge of the water. The influence of this distance was investigated by reducing it to $0.7 \mathrm{~m}$ and increasing it to $1.19 \mathrm{~m}$, with the resulting acceleration curves shown in fig. 13 . The three curves are all very similar, although the $0.7 \mathrm{~m}$ curve does begin to diverge slightly after about $0.027 \mathrm{~s}$. The influence of the depth was investigated by reducing it to $0.76 \mathrm{~m}$ and increasing it to $1.16 \mathrm{~m}$, fig. 14 . Again the curves are very similar with small divergence later in the analysis. No displacement boundary condition has been applied to the 
water boundaries, so any variation in results is due only to the change in inertial confinement from the size of the fluid domain. A water domain of $2.44 \mathrm{~m} \times 1.68 \mathrm{~m} \times 0.96 \mathrm{~m}$ with a $4 \mathrm{~cm}$ particle size provides a reasonable compromise between accuracy and computational cost for this model.

The overall deformation of the FE structure is consistent with the deformation observed in the experiment, figure 15. The test article shows a combination of plastic deformation, material failure and joint failure. The skin of the structure remains intact. No appropriate failure model was available to represent material failure in the FE model, so failure was ignored in the model. The locations of failure in the test are consistent with area of high plastic strain in the simulation results.

A direct comparison of the trolley acceleration results is difficult as the experimental results show that in the experiment the trolley and its supports were not fully rigid, unlike the representation of the trolley in the simulation. To correct this difference would require the addition of an FE model of the trolley which was not possible in this research. The peak acceleration levels are higher in the simulation but show a similar overall trend. The initial slope of the acceleration time curve from the simulation is higher than in the experiment as air is not included in the simulation. In the experiment there is an initial air cushion and a water-air mixture that can not be represented in the simulation. This difference is consistent with behaviour observed in other water impact simulations [21].

\subsection{CALM buoy model}

This model demonstrates the capability to perform a 3D simulation of wave interaction with a floating body. The problem consists of a 400 tonne cylindrical buoy with an outer diameter of $12 \mathrm{~m}$, moored to the sea bed with 6 mooring lines. 
The water component is modelled with 1,045,436 SPH particles, representing a domain $430.5 \mathrm{~m}$ long by $36 \mathrm{~m}$ wide and $28.5 \mathrm{~m}$ deep. The particle size is $0.75 \mathrm{~m}$. The domain length and particle size was selected following a 2D study investigating the effect of these parameters on the wave height measured at the buoy location. In practice the particle size of $0.75 \mathrm{~m}$ is too large, however approximately one million particles represented the practical upper limit for the number of particles that could be considered on the available computational resources, and the size was set once the size of the water domain was defined.

The buoy is modelled as a rigid body with defined mass and inertial properties. A rigid body is consistent with the test data, and a deformable body would require a higher fluid resolution. The buoy geometry is highly simplified as the particle size is too large to allow features such as the skirt to be included. The six mooring lines are each represented by 200 truss elements with the appropriate mass and stiffness properties. The mooring lines do not interact with the water as again, simulating this interaction would require the use of a far higher resolution of the water component to capture the hydrodynamic behaviour, impractical without a fully parallelized code and the use of a large super-computer. The cable interaction with the water is ignored in the simulation by excluding the nodes of the cable elements from the search for contact neighbours.

The state of the model at the start of the transient simulation is shown in fig. 16, showing the state of the model after dynamic relaxation has been used to solve for the initial equilibrium state. A detail view of the buoy and mooring lines is shown in fig. 17 .

In this model the computational cost of one SPH step is approximately three orders of magnitude greater than one FE step due to the number of SPH particles. The stable timestep size of the FE model is smaller than the SPH stable step size. As a consequence a subcycling algorithm was developed and implemented. This algorithm allows multiple FE steps per SPH step, with ten FE steps per SPH step used for this model, giving a very significant 
and necessary saving in computation time. Even so, 60 s of model time took approximately 6 days to run.

Simulation results for the buoy response to a $12 \mathrm{~m}$ extreme wave are shown in figure 18. The wave period in the analysis is $15 \mathrm{~s}$. The graph shows the vertical and longitudinal displacement of the buoy centre of gravity along with the pitch angle, with the arrival of the $12 \mathrm{~m}$ wave occurring at about $42 \mathrm{~s}$. The significant simplifications required to run this model on a scalar code prevent direct comparison with test data, however the overall motion of the buoy is sensible behaviour. In addition the simulation does show the flow of water over the top of the buoy as the wave passes, figure 19. As stated before the particle size is too large to provide adequate resolution for the local flow around the buoy. Refining the model is impractical without a parallel implementation of the coupled FE-SPH approach. This model will be revisited in the future once this is available.

\section{Conclusions}

Extreme waves can cause significant damage to ships and offshore structures. Currently there is no reliable theoretical or numerical technique available to predict the relevant loads and structural response.

The coupled Finite Element - Smoothed Particle Hydrodynamics approach has been developed for treating fluid-structure interaction problems where the structural response is potentially highly non-linear. This paper demonstrates that the approach can be extended to the treatment of extreme wave loading of floating structures though the addition of appropriate initial and boundary conditions.

A set of simulation results are shown to demonstrate that the approach allows:

- the correct behaviour of a floating body,

- the prediction of structural collapse under water loading, 
- 3D simulation of wave interaction with a floating body.

\section{Acknowledgement}

Part of the work presented in this paper was funded by the UK Engineering and Physical Sciences Research Council (EPSRC) under project GR/S95435/01.

\section{References}

[1] De Vuyst T, Vignjevic R, Campbell JC. Coupling between meshless and finite element methods. Int J Impact Engng 2005; 31: 1054-1064.

[2] Anghileri M, Castelletti L-ML, Francesconi E, Milanese A, Pittofrati M. Rigid body water impact - experimental tests and numerical simulations using the SPH method. Int J Impact Engng 2011; 38: 141-151.

[3] Groenenboom PHL, Cartwright BK. Hydrodynamics and fluid-structure interaction by coupled SPH-FE method. J Hydraulic Research 2010; 48 S1:61-73.

[4] Patel MH, Vignjevic R, Campbell JC. An SPH technique for evaluating the behaviour of ships in extreme ocean waves. Int J Maritime Engng 2009; 151: 39-47.

[5] Gingold RA, Monaghan J. Smoothed Particle Hydrodynamics: Theory and application to non-spherical stars. Mon Not R Astron Soc 1977; 181: 375-389.

[6] Lucy LB. A numerical approach to the testing of the fission hypothesis. Astron J 1977;82: 1013-1024.

[7] Monaghan JJ. Simulating free surface flows with SPH. J Comp Phys 1994; 110: 399406.

[8] Monaghan JJ. Scott Russell's wave generator. Phys Fluid 2000; 12: 622-630.

[9] Lo EYM, Shao S. Simulation of near-shore solitary wave mechanics by an incompressible SPH method. App Ocean Res 2002; 24: 275-286. 
[10] Lee E-S, Moulinec C, Xu R, Violeau D, Laurence D, Stansby P. Comparisons of weakly compressible and truly incompressible algorithms for the SPH mesh free particle method. J Comput Phys 2008; 227: 8417-8436.

[11] Hughes JP, Graham DI. Comparison of incompressible and weakly-compressible SPH models for free-surface water flows. J Hydraulic Research 2010; 48 S1:105-117.

[12] Gómez-Gesteira G, Cerqueiro D, Crespo C, Dalrymple RA. Green water overtopping analyzed with a SPH model. Ocean Engng 2005; 32: 223-238.

[13] Shao S, Ji C, Graham DI, Reeve DE, James PW, Chadwick AJ. Simulation of wave overtopping by an incompressible SPH model. Coast. Engng 2006; 53: 723-735.

[14] Wingate C, Dilts G, Crotzer L, Knapp C, Mandell D. Progress in Smooth Particle Hydrodynamics. Los Alamos National Laboratory Report LA-UR-98-300. 1998.

[15] Springel V. The cosmological simulation code GADGET-2. Mon Not R Astron Soc 2005; 364: 1105-1134.

[16] Ferrari A, Dumbser M, Toro EF, Armanini A. A new parallel SPH scheme for free surface flows. Computers \& Fluids 2009; 38: 1203-1217.

[17] Maruzewski P, Le Touzé D, Onger G, Avellan F. SPH high-performance computing simulations of rigid solids impacting the free-surface of water. J Hydraulic Res 2010; 48 S1:126-134.

[18] SPH European Research Interest Community (SPHERIC). http://wiki.manchester.ac.uk/spheric/index.php/Main_Page (last accessed December 2011).

[19] Buchner B, Bunnik THJ, Fekken G, Veldman AEP. A numerical study on wave run up on an FPSO bow. Presented at the 20th international conference on offshore mechanics and arctic engineering. Rio de Janeiro, Brazil. 2001. 
[20] Kleefsman KMT, Fekken G, Veldman AEP, Bunnik THJ, Buchner B, Iwanowski B. Prediction of green water and wave loading using a Navier-Stokes based simulation tool. Presented at the 2002 ASME Offshore Mechanics and Arctic Engineering conference, Oslo, Norway. 2002.

[21] De Vuyst T. Hydrocode modelling of water impact. PhD Thesis, Cranfield University, UK. 2003.

[22] Vignjevic R, De Vuyst T, Gourma M. On interpolation in SPH. Comput Model Eng Sci 2001; 2: 319-336.

[23] Vignjevic R, Campbell J, Jaric J, Powell S. Derivation of SPH equations in a moving referential coordinate system. Comput Methods Appl Mech Eng 2009; 198: 24032411.

[24] Lin, J.I. DYNA3D: A Nonlinear, Explicit, Three-Dimensional Finite Element Code for Solid and Structural Mechanics. User Manual. Lawrence Livermore National Laboratory, USA. 2004.

[25] Fekken, G. (2004) Numerical simulation of free-surface flow with moving rigid bodies. PhD Thesis, Groningen University, Netherlands. Available online at: http://irs.ub.rug.nl/ppn/260616745 (last accessed March 2008).

[26] Vigliotti, A. Metallic specimen test report, D.6.5.1.2. CAST (Crashworthiness of helicopters on water: design of structures using advanced simulation tools). EU Framework V project, Contract G4RD-CT1999-01728, CIRA, Italy. 2003. 


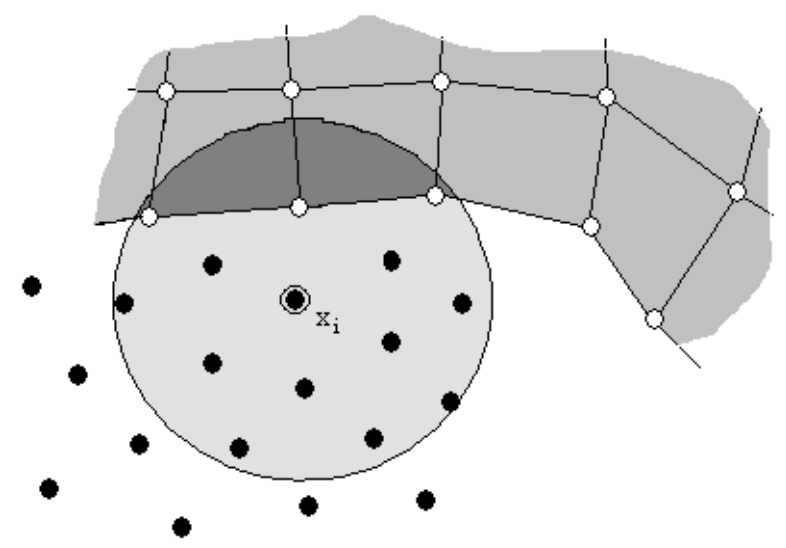

Fig. 1. Treatment of contact between SPH particles and FE mesh. SPH particle at point $\mathrm{x}_{i}$ interacts with all FE nodes within $2 h$, denoted by light grey circle. 


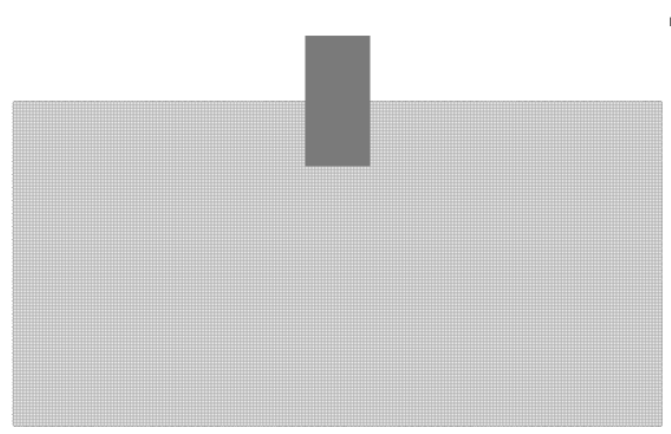

a
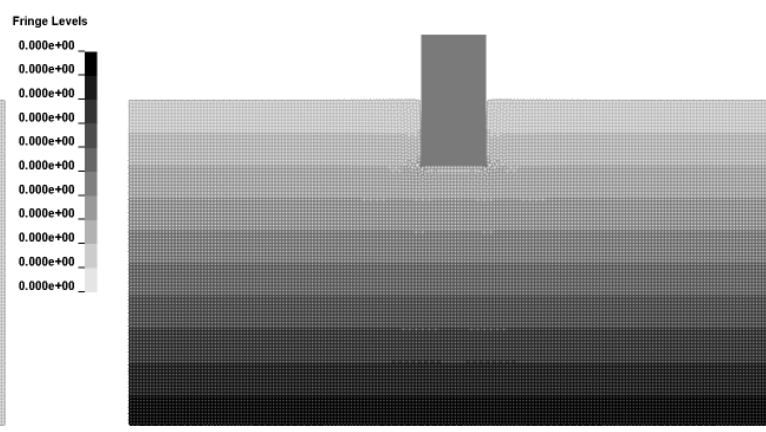

b

Fig. 2. Initial (a) and final (b) state of a dynamic relaxation analysis of a rectangular float.

Fringes of pressure (Mbar) are shown. 


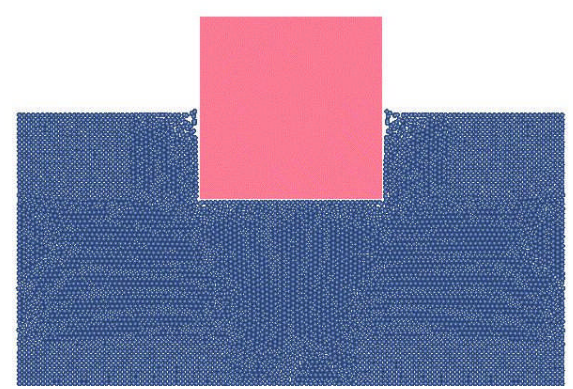

a

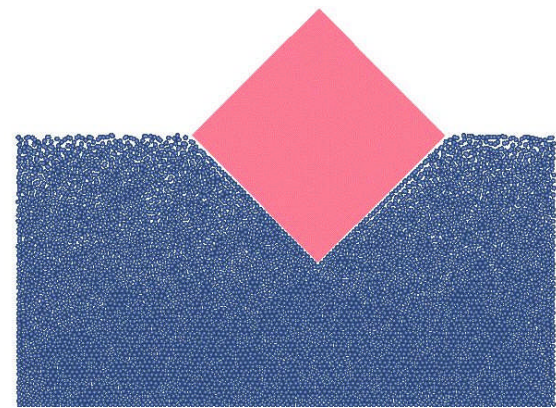

b

Fig. 3. Behaviour of a square float with density half the density of the fluid. a) Initial position. b) Final stable equilibrium position. 


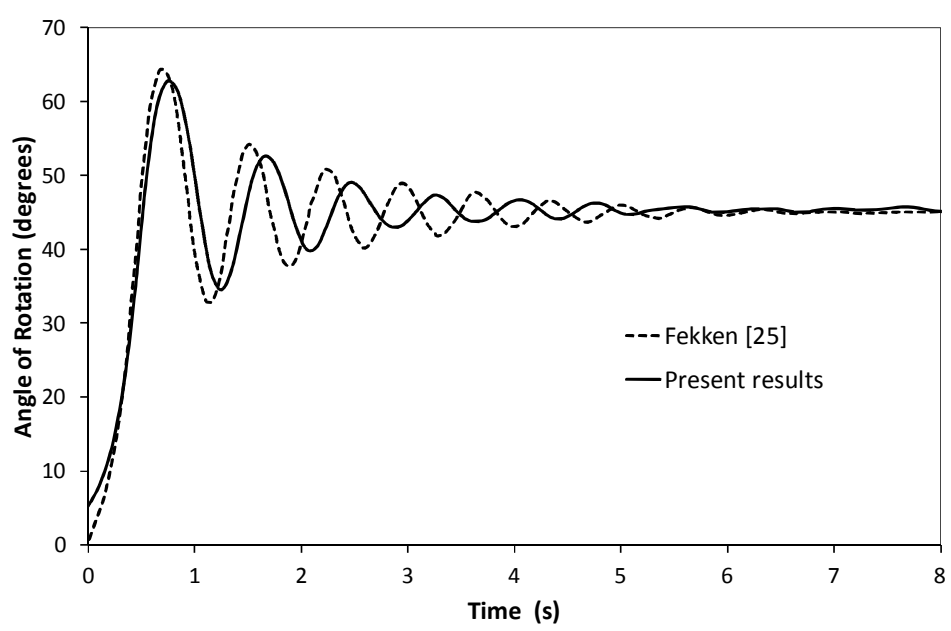

Fig. 4. Time history of rotation angle of square float with half the density of the fluid. 


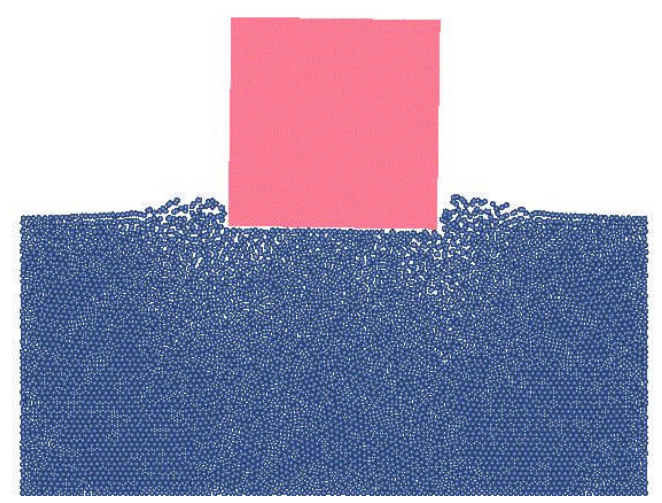

a

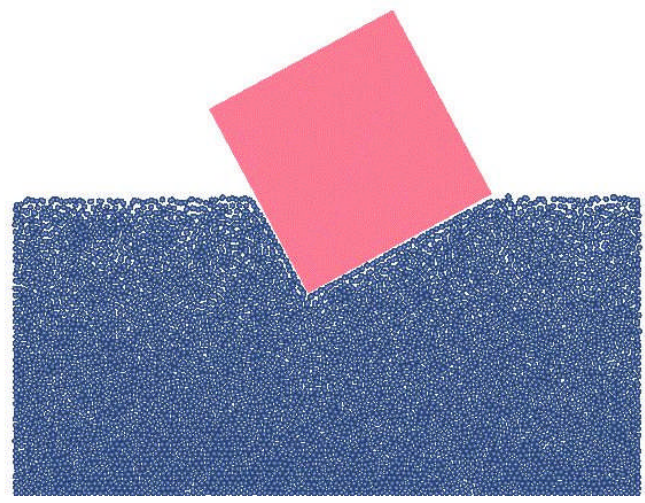

b

Fig. 5. Behaviour of a square float with density one quarter the density of the fluid. The float initially rises (a), The settles to the final stable equilibrium position (b). 


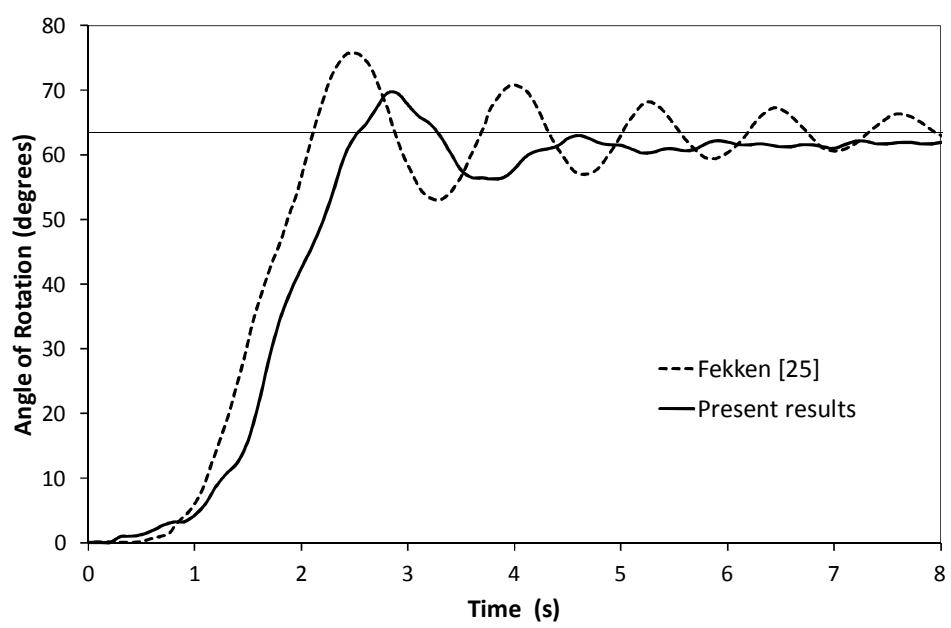

Fig.6. Time history for float with density one quarter the density of the fluid. The horizontal line on the graph marks the theoretical final angle of 63.4 degrees. 


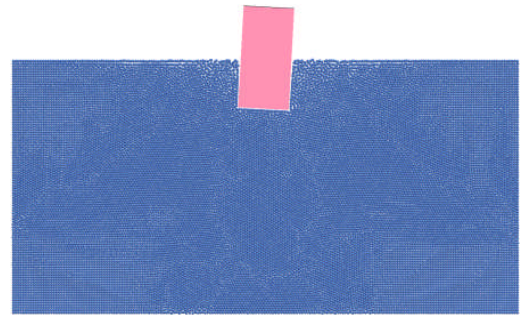

$0.6 s$

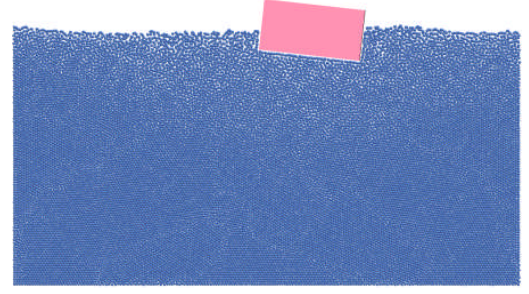

$1.8 \mathrm{~s}$

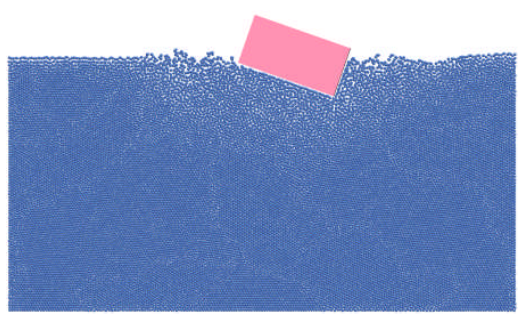

$1.2 \mathrm{~s}$

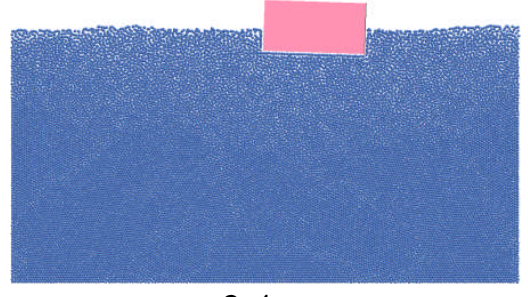

$2.4 \mathrm{~s}$

Fig. 7. Four images showing rotation of the rectangular float during the simulation 


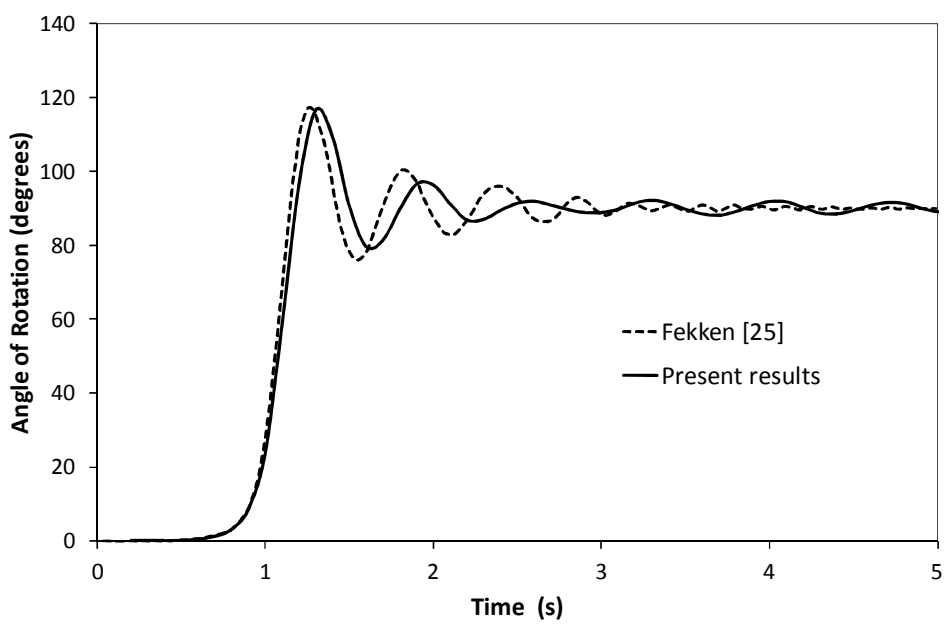

Fig. 8. Time history for rotation angle of rectangular float 


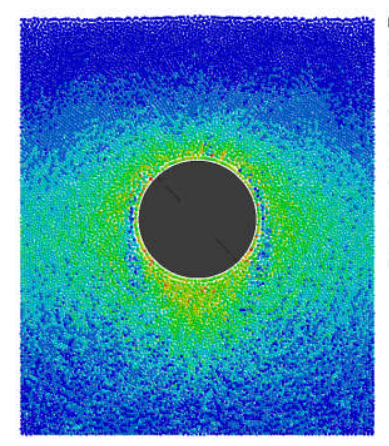

a

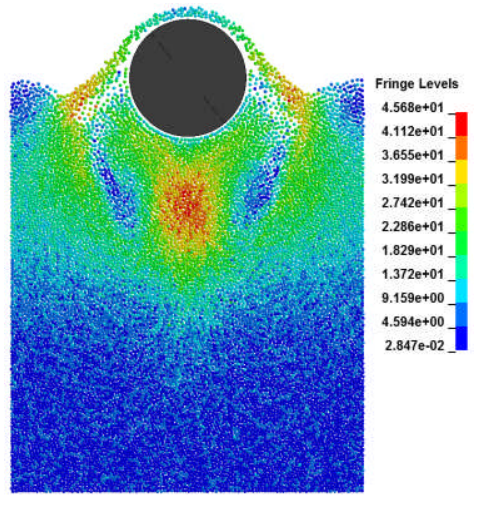

b

Fig. 9. Rising cylinder simulation results at time $1.5 \mathrm{~s} \mathrm{(a)} \mathrm{and} \mathrm{time} 3.0 \mathrm{~s}(\mathrm{~b})$ for particle spacing $0.05 \mathrm{~m}$ model. The fluid particles show resultant velocity $(\mathrm{m} / \mathrm{s})$. 


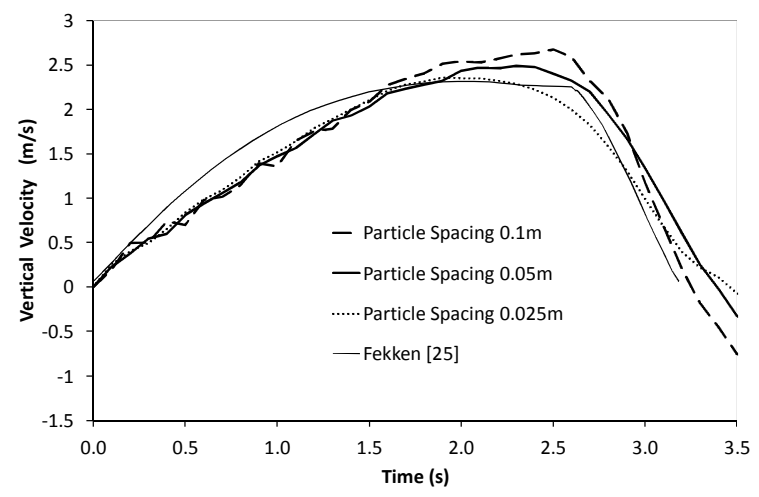

a

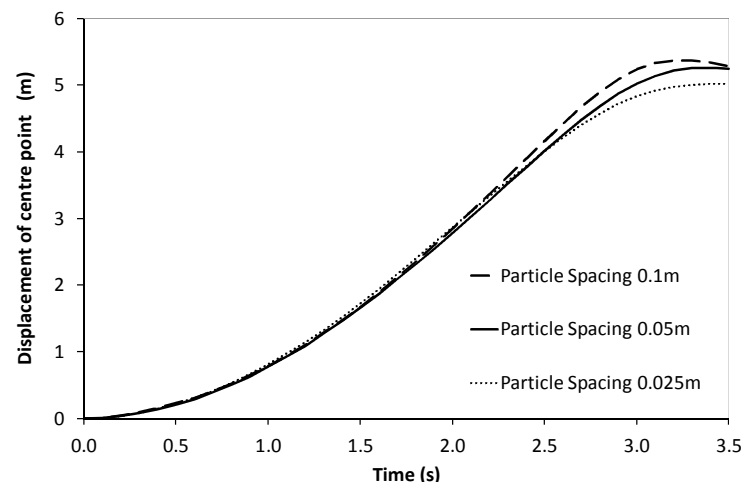

b

Figure 10: (a) Vertical velocity of rising cylinder with varying initial particle spacing, compared with published results. (b) Centre point position of rising cylinder. 


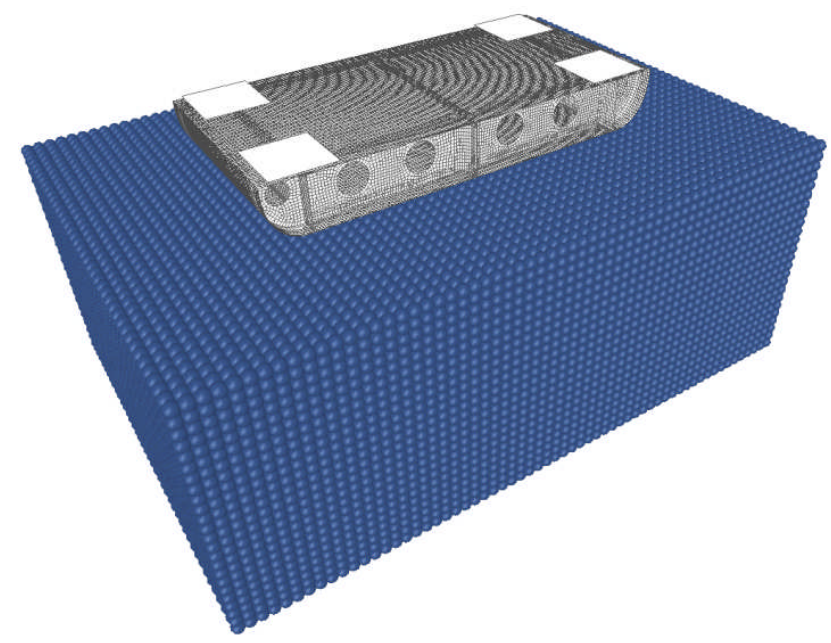

Fig.11. Initial model for helicopter sub-floor impact simulation. Particle size $4 \mathrm{~cm}$, water depth $0.96 \mathrm{~m}$, water extent $0.95 \mathrm{~m}$. 


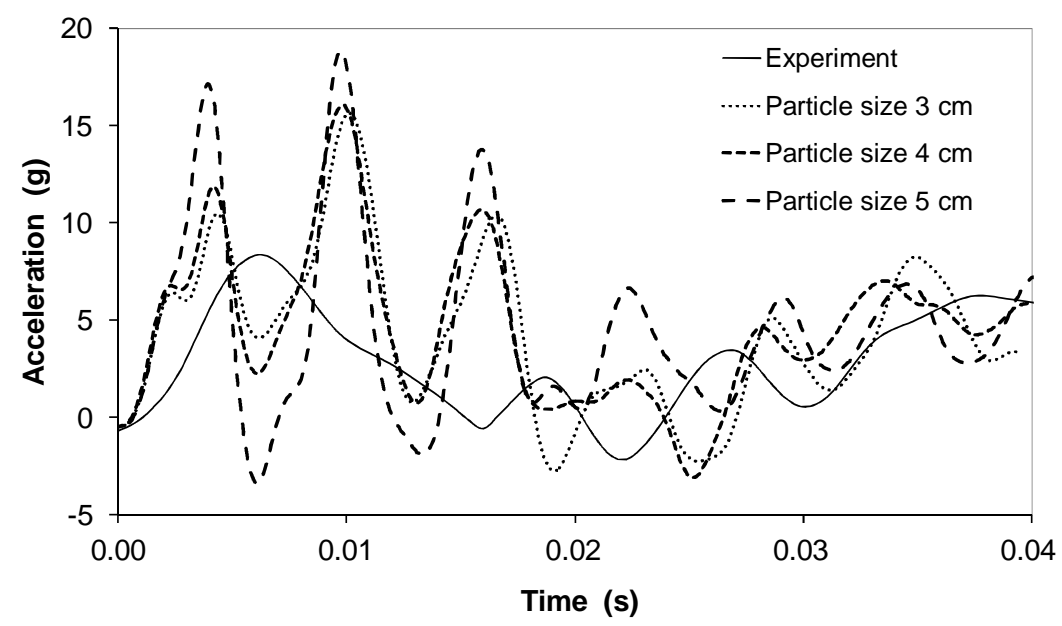

Fig. 12. Comparison of trolley accelerometer data to simulation results with varying extent of water domain. All curves have been filtered using an sae $180 \mathrm{~Hz}$ filter 


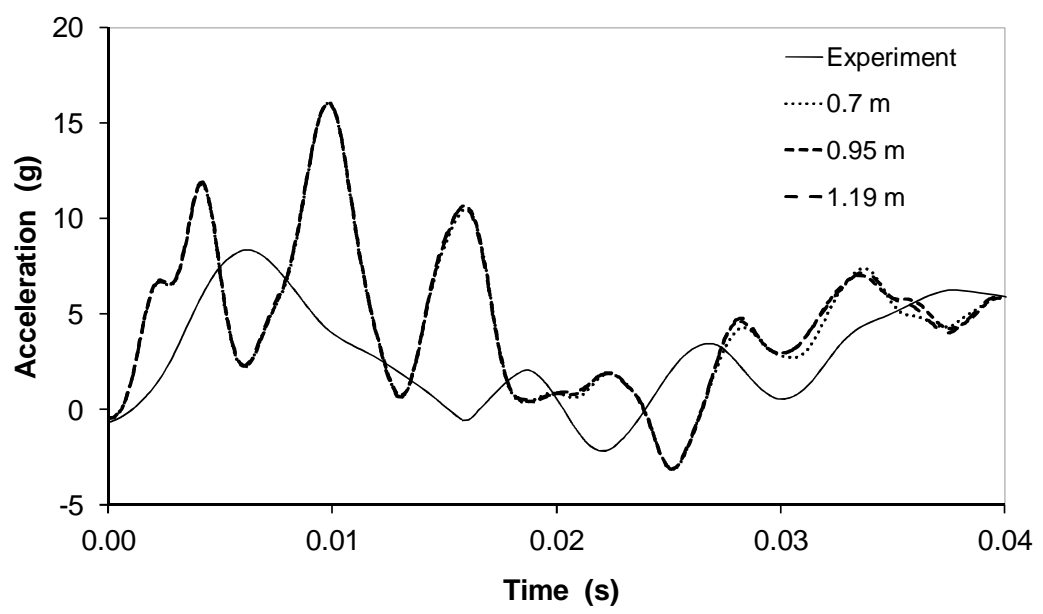

Fig. 13. Comparison of trolley accelerometer data to simulation results with varying extent of water domain. Value in legend refers to distance between structure and boundary of water domain. 


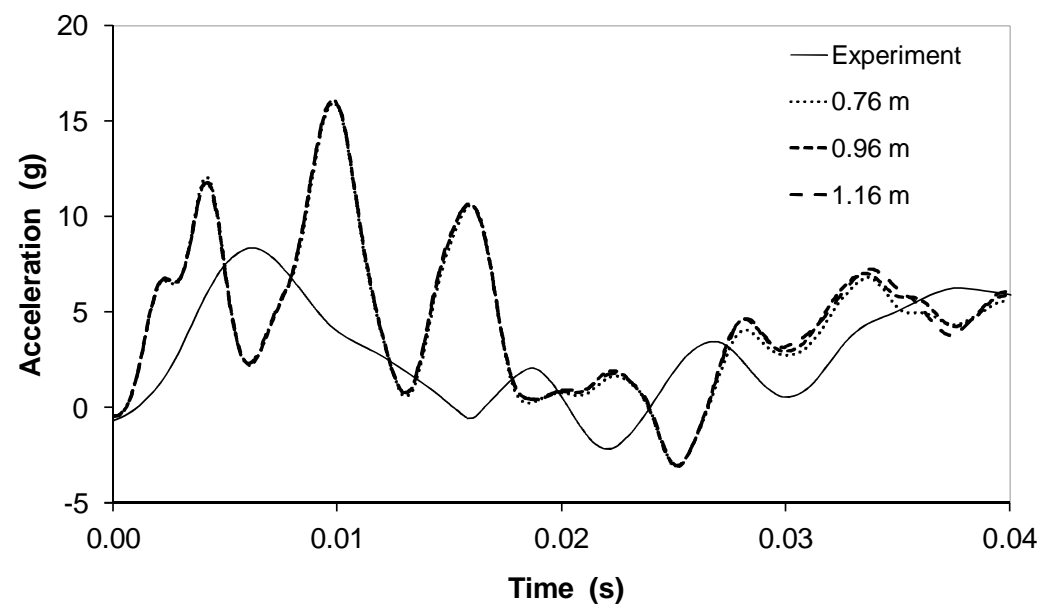

Fig. 14. Comparison of trolley accelerometer data to simulation results with varying depth of water domain. 


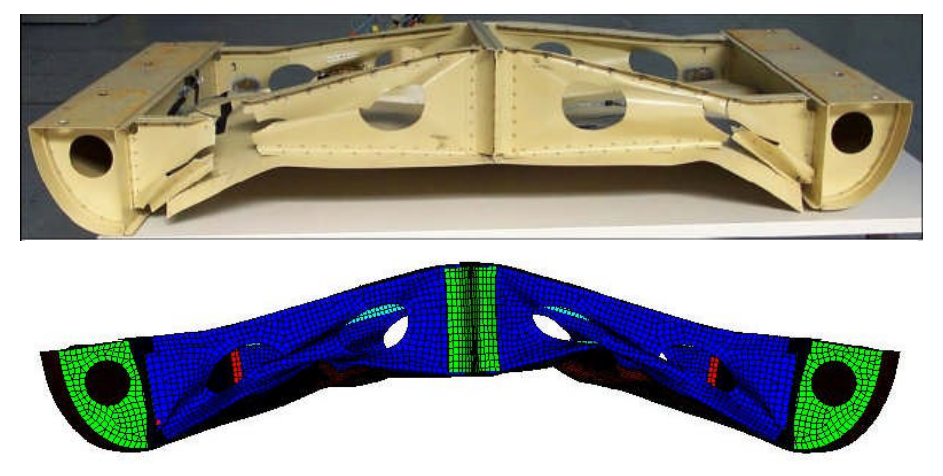

Fig. 15. Comparison of deformed shape of test article and FE mesh. 


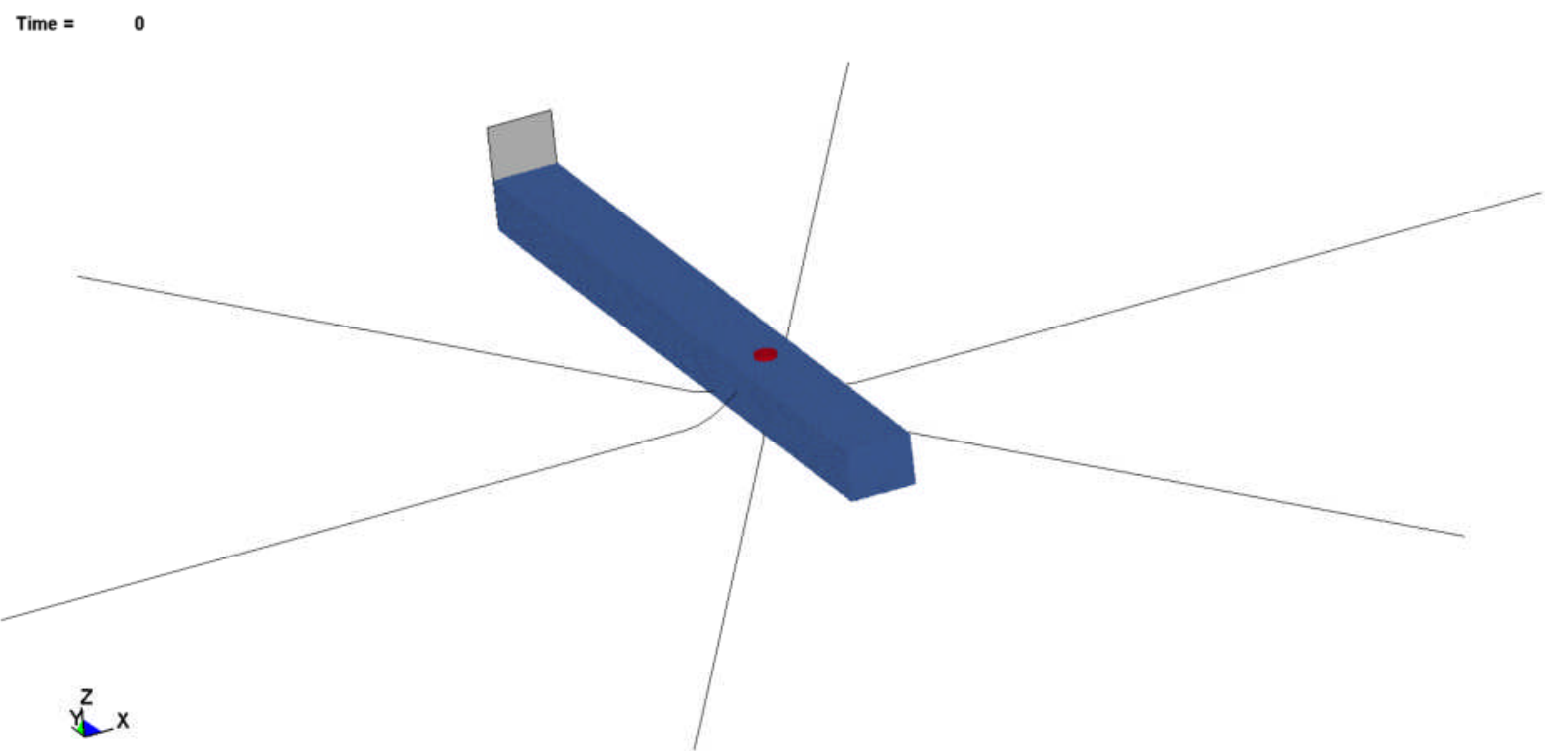

Fig. 16. View of complete buoy model at start of transient simulation 


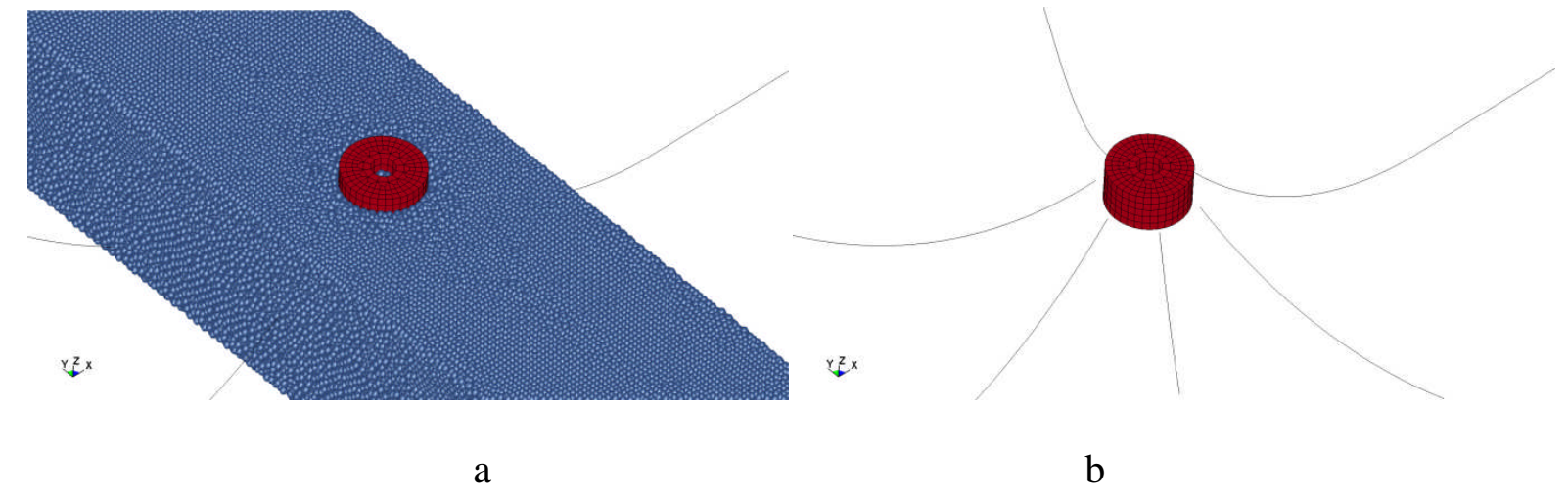

Fig. 17. a) Detail view of model in region of buoy. b) Detail view of buoy and cables with water removed. 


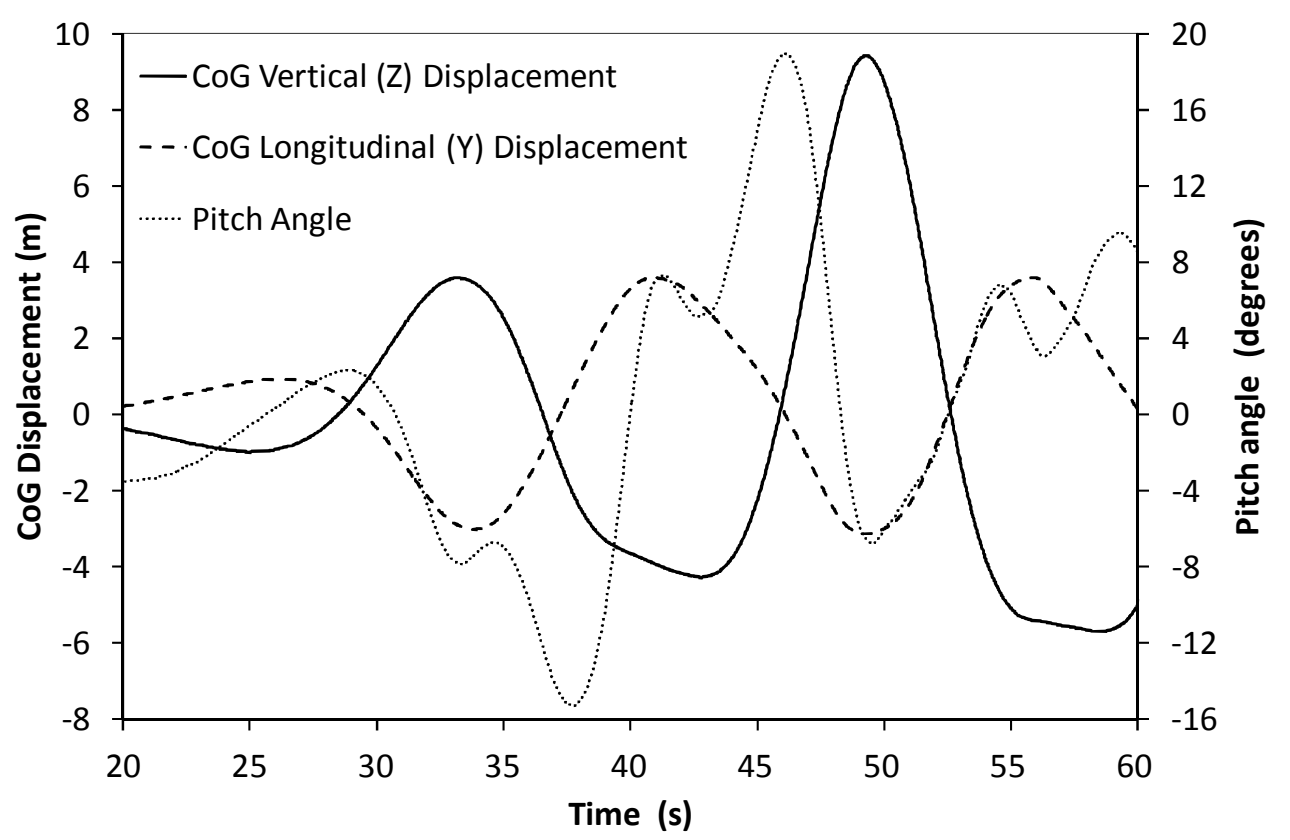

Fig. 18. Graph showing vertical longitudinal displacement of centre of gravity and pitch angle for buoy. 


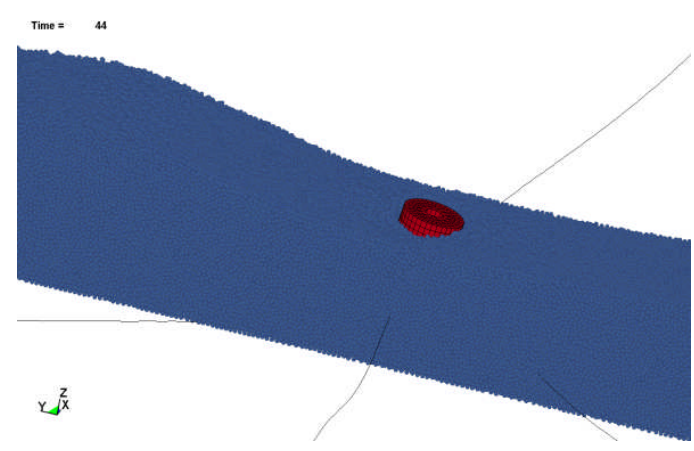

1

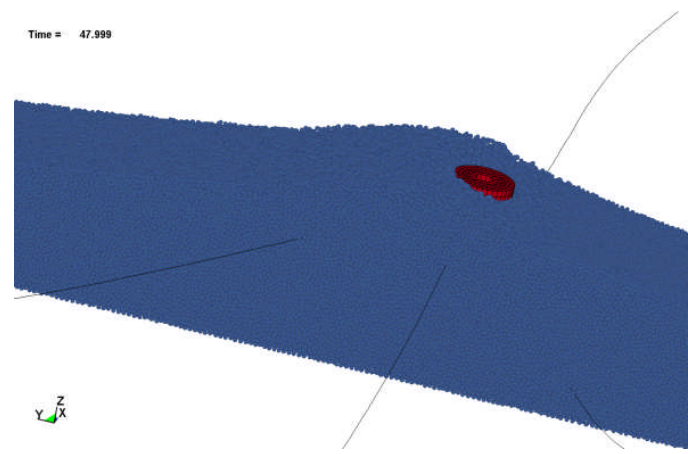

3

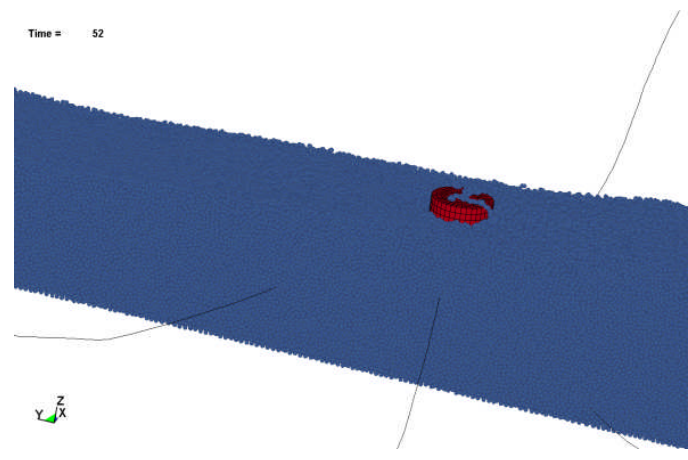

5

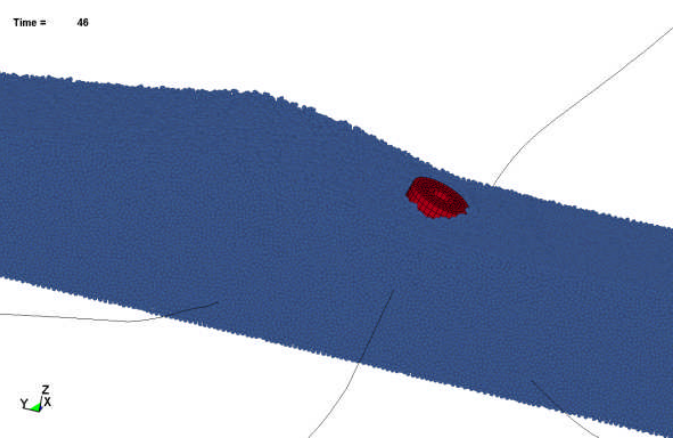

2

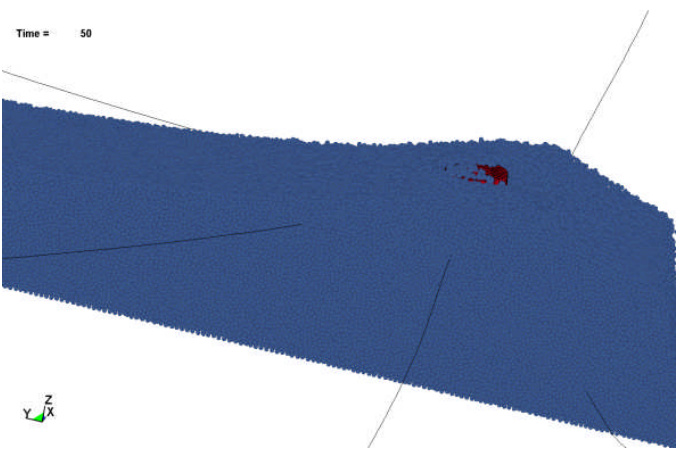

4

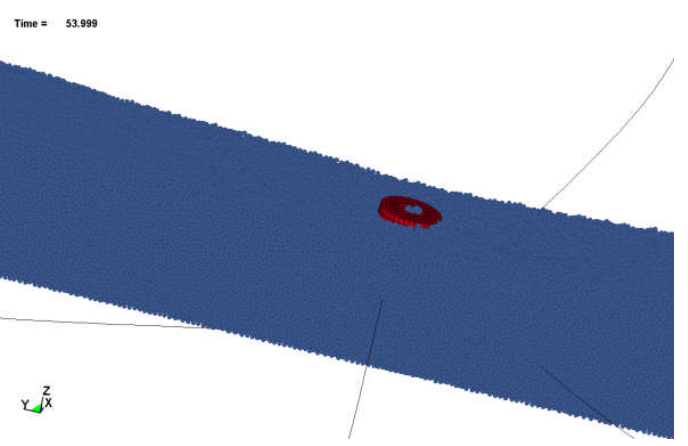

6

Fig. 19. Sequence showing the interaction of a $12 \mathrm{~m}$ high wave with a moored buoy. Note the wave overtopping that can be seen in images 4 and 5 . The time interval between the images is two seconds, with the initial image at $42 \mathrm{~s}$ and the final image at $52 \mathrm{~s}$. 
